671. Adolf Frnest und Heinr. Berger: Peroxydasen aus der Zuckerrtbo.

(Eingegangen am 13. November 1907.)

Mlit Bezug auf die Arbeiten von A. Bach und R. Chodat') sprach Julius Stoklasa die Vermutung aus, daß Peroxydasen wahrscheinlich in der Zuckerrube vorhanden seien. Auf seine Aufforderung unterzogen wir uns der Arbeit in dieser Richtung und zwar mit positivem Erfolge. Die ersten vorläufigen Versuche hat einer von uns direkt mit Rübenbrei, welcher aus den äußeren Partien der Zuckerrübe gewonnen war, ausgeführt.

Der Brei wurde zunächst der Einwirkung von 80-proz. Äthylalkohol ausgesetzt. Nach AbgieBen und Abpressen des Alkohols wurde der Brei einerseits mit Guajactinktur, andererseits mit Pyrogallıslösung und Wasserstoffhyperoxyd gepruft.

Obwohl die Guajactinktur eine Reaktion aut Peroxydasen lieferte, erachteten wir dieselbe (nach deo mit diesem ziemlich heiklen Reagenz gewonnenen Erfahrungen) nicht für maßgebend.

Die komparativen Versuche, bei welchen einerseits Pyrogalluslösung mit Wasserstoffhyperoxyd in destilliertem Wasser, ohne Hinzutun von Rübenbrei, andererseits unter Hinzufügung von Rübenbrei verwendet wurden, wiesen, je nach der Raschheit der Entstehung von Purpurogallin, auf die Gegenwart von Peroxydasen hin. Die Zersetzung des Wasserstoffhyperoxyds in der wäßrigen Lösung zeugte ron der gleichzeitigen Anwesenheit ron Katalase.

Um die Wirkung der Oxydasen aus Zuckerrübe besser studieren zu können, bemühten wir uns, sie zu isolieren, zum Zwecke der Ausscheiduvg des Einflusses der übrigen in der Zelle vorkommenden Enzyme.

Bei der Isolierung der Peroxydasen gingen wir teils nach der Auleitung Bachs vor, teils haben wir den Vorgang einigermaßen modifiziert.

Die von uns gewonnenen Präparate wiesen die Eigenschaften der Peroxydasen auf, und blob in einem Falle, in welchem zur Darstellung derselben junge Rüben verwendet wurden, zeigte das Präparat auch katalytische Eigenschaften, welche nach Losung der Substanz in Wasser und abermaliger Niederschlagung durch Alkohol-Äther vollständig verschwanden.

1) In diesen $>$ Berichten $\alpha$ publiziert. 
Darstellung der Peroxydasen aus Zuckerrübe.

$10 \mathrm{~kg}$ Kūbe (zur Herbstzeit gerade vom. Felde geholt) wurden uach erfolgter Waschung mit Wasser mittels eines Keibeisens abgerieben, so daß von jeder einzelnen Rübe bloß eine etwa $4 \mathrm{~cm}$ starke Schicht der obersten Partie zur Verwendung gelangte. Der Brei wurtle einerseits direkt mit 80-proz. $\bar{T}$ thylalkohol in einer Porzellanschale übergossen, andererseits in seiner zweiten Hallte unter einem 1)ruck ron ca. 300 Atmosphären ausgepreßt und der gewonnene Preßsaft in einen Glaszylinder gegossen, und hierauf die doppelte Menge 96-prozentigen Alkohols nachgefüllt.

Im ersten Falle wurde der Alkohol vom Rübenbrei nach 4 Tagen entSerut. Den Rest preßten wir bei niedrigeren Druck ab und üborgossen hernach den Brei mit 40-prozentigem ïthylalkohol und ließen ihn zum Zwecko der Extraktion 8 Tage stehen. Wer alkoholische Extrakt wurde hierauf von dem Brei, teils durch Filtration, teils durch Abpressen so schnell als möglich geschieden und bei $40-50^{\circ}$ abgedanpft, der konzentrierte Extrakt sodann

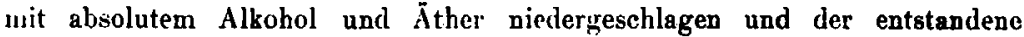
flockige (einen Stich ins Graue zeigende) Niederschlag durch Filtration von rler Flüssigkeit getrennt und bei $50^{\circ}$ getrocknet. Nachdem die Masse vollstăndig erhărtet war, zerrieben wir sie zu einem feinen Pulver und bewahrten dis letztere in einem hermetisch verschlossenen Gefäß im Exsiccator für weitere Versuche auf.

- In zweiten lialle, in welchen der geprebte Saft zur Verwendung gulangte, wurde der Alkohol mit dem Wasser von dem schmierigen Teile abgeschieden und der nach 24 Stunden sich absetzonde Rest einer Extraktion mit 40-prozentigem Alkohol unterworien. Nach 8 T'agen wurde die alkoholische Liisung abfiltriert, konzentriert und getrocknet, ganz so, wie wir früher angegeben hahen.

Wir gewannen auf diese Weise $2.3 \mathrm{~g}$ rohen, gegeniiber $1.7 \mathrm{~g}$ des vorgenannten Präparates.

Wiewobl diese Differenzen nicht bedeutend sind, scheint es doch, daß 3 von dem erwähnten Rubenbrei etwas von der Substanz direkt in den Saft überging. Die so gewonnenen $4 \mathrm{~g}$ der Substanz wurden geprüft und erwiesen sich als Peroxydasen. $0.2 \mathrm{~g}$ der Substanz wurden in $20 \mathrm{ccm}$ destillierten Wassers aufgelöst, filtriert uud $20 \mathrm{ccm}$ 1-prozentigen Wasserstoffhyperoxyds in Wasser mit $2 \mathrm{~g}$ Pyrogallol hinzugefügt. Parallel wurde derselbe Versuch nur mit dem Unterschiede durchgefïhrt, daß keine Peroxydasen hinzugefügt wurden; an ihrer Statt verwendeten wir eine Lösung ron $2 \mathrm{~g}$ Pepton, und schließlich unternahmen wir einen analogen dritten Versuch, wobei zu $20 \mathrm{ccm}$ 1-prozentiger Wasserstoffhyperoxydlösung nit $2 \mathrm{~g}$ Pyrogallol $20 \mathrm{ccm}$ destillierten Wassers ohne irgendwelche Zutat gefügt wurde. Alle drei Versuche unternahmen wir unter Einhaltung dersellien äußeren Bedingungen (Temperatur, Licht usw.). Nach Verlauf von etwa 5 Minuten zeigte die Lüsung, in welche Peroxydasen getan worden waren, eine intensiv rote, in dunkelrot übergehende Verfär- 
bung, wäbrend die übrigen beiden Lösungen in derselben Zeit ohne Veründerung blieben.

Nach dieser qualitatiren Prúfung betreffs der Aktivität der Peroxydasen und AusschluB der Gegenwart der Katalasen und Oxydasen untersuchten wir die Aktivität quantitativ, wobei uns das ausgeschiedene Purpurogallin als Indicator diente. Bei diesem Vorgang trachteten wir, die Wirkung aller drei Faktoren, die bei der Bildung ron Purpurogallin ron Einfluß sind, zu erkennen und zwar der Peroxydasen, des Wasserstoffhyperoxyds und des Pyrogallols, indem wir alle äußeren Einflüsse durch Einbaltung derselben Bedingungen bei Durchfïhrung der Versuche ausschlossen. Das entstandene Purpurogallin wurde auf einem getrockneten und gewogenen Filter gesammelt.

\section{Versuchs reihe.}

Bei steigender Menge der Peroxydasen in einer konstanten Menge Wissecrstoffhyperoxyds und Pyrogallols; Parallelversuche in Abwesenheit von l'eroxydasen, Finhaltung derselben Konzentration auch bei den weiteren Versuchen.

Innerhalb 24 Stunden.

\begin{tabular}{|c|c|c|c|c|}
\hline $\begin{array}{c}\text { Peroxy- } \\
\text { dasen } \\
\end{array}$ & $\begin{array}{c}\text { 1-proz. } \\
\mathrm{H}_{2} \mathrm{O}_{2} \text {-Lösung } \\
\text { in Wasser } \\
\text { ccm } \\
\end{array}$ & Pyrogallol & $\begin{array}{c}\text { Purpuro- } \\
\text { gallin } \\
g \\
\end{array}$ & $\begin{array}{l}\text { Unter denselben. } \\
\text { Bedingungen, ohne Hinzil- } \\
\text { fügen von Peroxydasen } \\
\text { entstandenes Purpurogallin }\end{array}$ \\
\hline $\begin{array}{l}0.15 \\
0.1 \\
0.15 \\
0.2\end{array}$ & $\begin{array}{l}15 \\
15 \\
15 \\
15\end{array}$ & $\begin{array}{l}1.5 \\
1.5 \\
1.5 \\
1.5\end{array}$ & $\begin{array}{l}0.0191 \\
0.0229 \\
0.0251 \\
0.0298\end{array}$ & 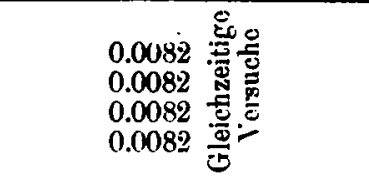 \\
\hline
\end{tabular}

II. Versuchsreihe.

Bei konstanter Menge der Peroxydasen und des Wasserstoffhyperoxyds, bei steigender Menge des Pyrogallals. Parallelversuche in Abwesenheit von Peroxydesen.

Innerbalb 24. Stunden.

\begin{tabular}{|c|c|c|c|c|}
\hline $\begin{array}{c}\text { Peruxy- } \\
\text { dasen } \\
\mathrm{g} \\
\end{array}$ & $\begin{array}{c}\text { 1-proz. } \\
\mathrm{H}_{2}\left(\mathrm{C}_{2}-\text { Lösung }\right. \\
\text { in Wasser } \\
\text { ccm }\end{array}$ & $\begin{array}{c}\text { Pyrogallol } \\
8 \\
\end{array}$ & $\begin{array}{c}\text { Purpuro- } \\
\text { gallin } \\
\text { g }\end{array}$ & $\begin{array}{l}\text { Unter denselben } \\
\text { Bedingungen, ohne Hinzu- } \\
\text { fügen von Peroxydasen } \\
\text { entstandenes Purpurogallin }\end{array}$ \\
\hline $\begin{array}{l}0.3 \\
0.3 \\
0.3 \\
0.3\end{array}$ & $\begin{array}{l}20 \\
20 \\
20 \\
20\end{array}$ & $\begin{array}{c}2 \\
2.5 \\
3 \\
8.5\end{array}$ & $\begin{array}{l}0.0882 \\
0.0866 \\
0.0820 \\
0.07 .57\end{array}$ & $\begin{array}{l}0.000 \\
0.0117 \\
0.0119 \\
0.0129 \\
0.0129\end{array}$ \\
\hline
\end{tabular}


JII. Versuchsreihe.

Bei konstanter Menge der Peroxyclasen und des Pyrogallols und steigender Menge des Wasserstoffhyperoxyds. Parallelversuche ohne Peroxydase.

Innerhalb 24 Stunden.

\begin{tabular}{|c|c|c|c|c|}
\hline $\begin{array}{c}\text { Peroxy- } \\
\text { dascn } \\
r \\
\end{array}$ & $\begin{array}{c}\text { 1-proz. } \\
\mathrm{H}_{2} \mathrm{O}_{2}-\text { Lousung } \\
\text { in Wasser } \\
\text { ccm }\end{array}$ & $\begin{array}{c}\text { Pyrogallor } \\
\mathrm{g}\end{array}$ & $\begin{array}{l}\text { Purpuro- } \\
\text { gallin } \\
\mathrm{g} \\
\end{array}$ & $\begin{array}{l}\text { Unter denselben } \\
\text { Bedingungen, ohne Hinzu- } \\
\text { fügen von Peroxydasen } \\
\text { entstandenes Purpurogallin }\end{array}$ \\
\hline $\begin{array}{l}1 . .2 \\
1.2 \\
0.2 \\
(1.2\end{array}$ & $\begin{array}{l}20 \\
30 \\
40 \\
50\end{array}$ & $\begin{array}{l}1.5 \\
1.5 \\
1.5 \\
1.5\end{array}$ & $\begin{array}{l}0.0267 \\
0.0298 \\
0.0458 \\
0.0497\end{array}$ & 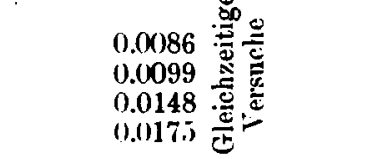 \\
\hline
\end{tabular}

Zur Bereitung des weiteren Präparats wurdeu $12 \mathrm{~kg}$ junger Rüben (gegen Ende Juli ausgehoben) verwendet. Das im Wege der Extraktion gewonnene Präparat (da eine Presse nicht zur Disposition war) wurde dahin geprüft, ob nur Oxydasen rorhanden waren und nachstehende Untersuchungen durchgeführt:

$A$ uf Oxydasen: $0.1 \mathrm{~g}$ roher Peroxydasen wurden in $10 \mathrm{ccm}$ Wasser gelüst und diese Lösung zur Pyrogallollösung zugegeben, $2 \mathrm{~g}$ in $20 \mathrm{erm}$ Wasser. Purpurogallin wurde selbst nach drei 'Tagen nicht ausgeschieden. 1)ie Flïssigkeit fărbte sich nur braun.

Katalase: $0.1 \mathrm{~g}$ roher Peroxydasen wurden in $10 \mathrm{ccm}$ Wasser gelöst, filtriert und im volumetrischen Apparate geprüft; mit 1-prozentiger Wasserstoffhyperoxydlossung entwickelte sich Gas, was das Vorhandensein ron Katalase beweist.

A m ylase: $100 \mathrm{ccm}$ 2-prozentiger sterilisierter Stärkckleister wurden mit $\because \mathrm{g}$ Peroxydasen in $10 \mathrm{ccm}$ Wasser gemengt und durch 24 Stunden bei $30^{\prime \prime}$ in Thermostaten gebalten. Der Kleister reduzierte Fehlingsche läsung nach dem Versuche nicht, Amylase war also nicht vorhanden.

Invertase: Die Probe wurde in der üblichen Weise mit 2-prozentiger Saccharoselösung durchgeführt mit negativem Erfolge.

Emulsin: $0.2 \mathrm{~g}$ roher Peroxydasen wurden in $10 \mathrm{ccm}$ Wasser gelöst und zur Lōsung Amygdalin (1:20) in Wasser hinzugetan. Lis wurde weder ein Geruch nach Benzaldehyd, noch eine Entwicklung von Cyanwasserstofi wahrgenommen. Die Lösung reduzierte auch $\mathrm{F}$ ehlingsche Lósung nicht.

Proteolytische Enzyme: Die Lösung von Peroxydasen löste in der Hitze koaguliertes Eiweis nicht. Der Versuch wurde bei einer Temperatur von $30^{\circ}$ unter Hinzufügung von 3 Tropfen Toluol ausgeführt.

Mit Rücksicht auf die Cegenwart der Katalase wurde ihre Wirkung geprüft. $Z u$ diesem Zweck wurden $z$ wei Nitrometer verwendet, welche mit 2-prozentiger Wasserstoffhyperoxydlösung gefüllt wurden In das eine wurde ein abgewogenes Quantum roher Peroxydase in 
wäBriger Lösung binzugefägt, in das zweite gleichzeitig dieselbe Menge Pepton, in dem gleichen Quantum Wasser gelöst. Das entbundene Gas (Sauerstoff) haben wir gleichzeitig in den gleichen Zeitintervallen gemessen.

Auf den normalen Barometerdruck und $0^{0}$ Temperatur berechnet.

\begin{tabular}{c|c|c|c|c}
\hline $\begin{array}{c}\text { Verwendete Menge } \\
\text { der Peroxydasen } \\
\text { in 10ccm destilliert. } \\
\text { Wassers }\end{array}$ & Dersuchs & $\begin{array}{c}\text { Frei ge- } \\
\text { Wordener } \\
\text { Sauerstoff }\end{array}$ & $\begin{array}{c}\text { Bei denselben Ver- } \\
\text { haltnissen unter } \\
\text { Anwendung von } \\
\text { Pepton frei geword. } \\
\text { Sauerstoff } \\
\text { ccm }\end{array}$ & $\begin{array}{c}\text { Durch katalyt. } \\
\text { Wirkúng } \\
\text { der Peroxydasen } \\
\text { frei gewordener } \\
\text { Sauerstoff }\end{array}$ \\
\hline & Minuten & & & \\
0.1 & 15 & 1.98 & 0.27 & 1.71 \\
0.15 & 15 & 2.88 & 0.27 & 2.61 \\
0.2 & 15 & 3.60 & 0.18 & 3.42 \\
0.1 & 30 & 2.79 & 0.27 & 2.52 \\
0.2 & 30 & 4.77 & 0.36 & 4.41
\end{tabular}

Der Rest dieses Präparates, von dem ursprünglich $5.705 \mathrm{~g}$ rorhanden waren, wurde in Wasser aufgelöst und mit absolutem Alkohol und Äther nochmals niedergeschlagen, bei $40^{\circ}$ getrocknet und neuerlich untersucht. Katalytische Wirkungen wurden jetzt nicht beobachtet. Bei Hinzufügung ron Pyrogallollosung und Wasserstoffhyperoxyd zur wäbrigen Losung hat die so neugewonnene Substanz Purpurogallin ausgeschieden, welche sodann gewogen wurde.

\section{Versuchsreihe.}

Bei konstanten Mengen Wasserstoffsuperoxyds und Pyrogallols bei steigenden Peroxydasenquantitāten.

\begin{tabular}{|c|c|c|c|c|}
\hline $\begin{array}{c}\text { Verwendete Menge } \\
\text { der Perorydasen } \\
\text { in } 10 \text { ccm destilliert. } \\
\text { Wassers } \\
\text { g } \\
\end{array}$ & $\begin{array}{c}\text { 1-proz. } \\
\text { Wasserstoff- } \\
\text { hyperoxyd- } \\
\text { lösung } \\
\text { ccm }\end{array}$ & $\begin{array}{l}\text { Pyro- } \\
\text { gallol } \\
\mathbf{8} \\
\end{array}$ & $\begin{array}{c}\text { Purpuro- } \\
\text { gallin } \\
\mathbf{g}\end{array}$ & $\begin{array}{c}\text { Purpurogallin im Parallel- } \\
\text { versuche ohne Hinzu- } \\
\text { fügung von Peroxydasen } \\
\mathbf{g}\end{array}$ \\
\hline $\begin{array}{l}0.1 \\
0.2 \\
0.25 \\
0.3\end{array}$ & $\begin{array}{l}30 \\
30 \\
30 \\
30\end{array}$ & $\begin{array}{l}2 \\
2 \\
2 \\
2\end{array}$ & $\begin{array}{l}0.0098 \\
0.0142 \\
0.0255 \\
0.0276\end{array}$ & 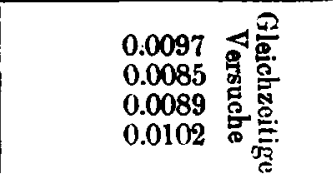 \\
\hline
\end{tabular}

Ein weiteres Präparat, ebenso gewonnen aus $15 \mathrm{~g}$ Samenrübe im Gewiehte von $6.52 \mathrm{~g}$, enthielt auch keine Oxydasen, Katalasen, Anylase, Frvertase, Emulsin oder proteolytische Enzyme und zeigte die Eigenschaften der Peroxydasen. 
V. Versuchsreihe.

Bei konstanten Quantitäten Pyrogallol, Wasserstoffhyperoxyd und steigenden Mengen von Peroxydasen.

\begin{tabular}{|c|c|c|c|c|}
\hline $\begin{array}{c}\text { Proxydasen in } \\
10 \mathrm{ccm} \\
\text { clest. Wassers } \\
g\end{array}$ & $\begin{array}{c}1-\mathrm{proz}_{2} \\
\mathrm{H}_{2} \mathrm{O}_{2} \\
\mathrm{ccm}\end{array}$ & $\begin{array}{c}\text { Pyro- } \\
\text { gallol } \\
\quad r \\
\end{array}$ & $\begin{array}{c}\text { l'urpuro- } \\
\text { gallin } \\
g \\
\end{array}$ & $\begin{array}{c}\text { Ohne Hinzufügung von } \\
\text { Peroxydasen } \\
\text { entst. Purpurogallin } \\
\mathrm{g}\end{array}$ \\
\hline $\begin{array}{l}0.105 \\
0.1 \\
0.15 \\
0.2 \\
0.3 \\
0.4\end{array}$ & $\begin{array}{l}20 \\
20 \\
20 \\
20 \\
211 \\
20\end{array}$ & $\begin{array}{l}2 \\
2 \\
2 \\
2 \\
2 \\
2 \\
2\end{array}$ & $\begin{array}{l}0.1) 156 \\
0.0282 \\
0.0345 \\
0.0367 \\
0.0473 \\
0.04499\end{array}$ & $\begin{array}{l}0.0123 \\
0.0147 \\
0.0103 \\
0.0114 \\
0.0113 \\
0.0135\end{array}$ \\
\hline
\end{tabular}

Aus dem Angeführten geht hervor:

1. die Zellen der Zuckerrüben enthalten Peroxydasen;

-2. diese Peroxydasen (Rohprodukt) lassen sich isolieren unter Ausscheidung der Wirksamkeit anderer Enzyme mittels Alkohols und ïthers;

3. die Menge des durch die Wirkung der Peroxydasen entstehenden Purpurogallins steigt mit der wachsenden Menge der angewendeten Peroxydasen und des Wasserstoffhyperoxyds, während die steigende Menge des Pyrogallols ihre Wirksamkeit lähmt. (Bei Berücksichtigung der Grenzen, innerhalh welcher unsere Versuche vich bewegten.)

\section{Teil.}

Ein weiteres Präparat wurde aus $50 \mathrm{~kg}$ Samenrüben nach der bereits früher angegebenen Methode hergestellt (Bach). Die Prüfung der Aktivität des Präparats, von dem im ganzen $3.4482 \mathrm{~g}$ gewonnen worden waren, erfolgte durch Wägung des aus der Lösung des Pyrogallols in Wasserstofihyperoxyd durch die Finwirkung von Peroxydasen ausgefällten Purpurogallins.

$0.2 \mathrm{~g}$ Peroxydasen scheiden aus der Lösung ron $2 \mathrm{~g}$ Pyrogallol in $20 \mathrm{~cm}$ Wasser und $90 \mathrm{ccm}:-$-prozentigen Wasserstoffhyperoxyds innerhalb 12 Stunden 0.037.2 g Purpurogallin aus, während ohne Peroxydasen unter densellien Bedingungen nur $0.0092 \mathrm{~g}$ Purpurogallin ausgeschieden wurden.

Es läßt sich mithin das Präparat als aktives zu weiteren Versuchen verwenden. Zur Prüfung dieses Präparats wurde die Methode voll W. Palladin angewendet ${ }^{1}$ ).

Es wurde $1 \mathrm{~g}$ des Peroxydasepräparats in $25 \mathrm{ccm}$ Wassers aufgelöst und diese Lösung in $\$ 0 \mathrm{ccm}$ 20-prozentiger, wäbriger Lösung von

"W. Palladin, Ztschr. f. physiol. Chem. 47, 407. 
Pyrogallol gegossen und zwar in einen Kolben, der dem Apparat von Stoklasa zur Prúfung der enzymatischen Tätigkeit betrefis der Kohlendioxydproduktion angeschlossen war.

Temperatur $20^{\circ}$.

\begin{tabular}{c|c}
\hline \hline $\begin{array}{c}\text { Dauer des Versuches } \\
\text { in Stunden }\end{array}$ & $\begin{array}{c}\text { Menge des produzierten } \\
\mathrm{CO}_{2} \text { in mg }\end{array}$ \\
\hline 7 & 21.6 \\
20 & 34.9 \\
6 & 7.3 \\
16 & 0.1 \\
\hline 49 & 63.9
\end{tabular}

Hinzufügen von $80 \mathrm{ccm}$ 30-prozentigem $\mathrm{H}_{2} \mathrm{O}_{2}$.

Temperatar $20^{\circ}$.

\begin{tabular}{r|r}
24 & 285.6 \\
24 & 30.4 \\
24 & 13.7 \\
\hline 72 & 329.7
\end{tabular}

Der Versuch wurde mit einer neuen Partie des Prüparates wiederholt. Es wurde $1 \mathrm{~g}$ Peroxydase abgewogen.

\begin{tabular}{c|r}
8 & 24.0 \\
14 & 14.2 \\
24 & 7.1 \\
\hline 46 & 45.3
\end{tabular}

Hinzulügung von $80 \mathrm{cem}$ 3-prozentigem $\mathrm{H}, \mathrm{O}_{2}$.

\begin{tabular}{c|r}
24 & 192.4 \\
24 & 44.5 \\
24 & 17.4 \\
24 & 9.8 \\
\hline 96 & 264.1
\end{tabular}

Der Rest des Peroxydasepräparats wurde bei einer Temperatur. von $140^{\circ}$ durch 8 Stunden getrocknet, worauf genau so vorgegangen wurde, wie bei dem vorbeschriebenen Versuch.

Das Präparat wog nach der Trocknung $1.0257 \mathrm{~g}$ und löste sich nicht mehr so gut im Wasser. (Vor dem Trocknen wog es 1.1067 g.);

\begin{tabular}{c|c}
\hline $\begin{array}{c}\text { Dauer des Versuches } \\
\text { in Stunden }\end{array}$ & $\begin{array}{c}\text { Menge des produzierten } \\
\mathrm{CO}_{2} \text { in } \mathrm{mg}\end{array}$ \\
\hline 6 & 4.0 \\
24 & 4.8 \\
\hline 30 & 8.8
\end{tabular}

Hinzufügung von $80 \mathrm{ccm}$ 3-prozentigem $\mathrm{H}_{2} \mathrm{O}_{2}$.

\begin{tabular}{c|c}
24 & 36.0 \\
24 & 10.8 \\
\hline 48 & 46.8
\end{tabular}


Lin weiteres Peroxydasepräparat wurde aus Radieschen bereitet, und zwar aus $10 \mathrm{~kg}$ junger Radieschen, zu dem Zweck, um die Wirkung des Radieschenpräparats') mit dem Präparat aus Zuckerrübe vergleichen zu können.

Zur Ilerstellung des Präparats wurde nur die obere Partie der Radieschen in einer Tiefe ron $3 \mathrm{~mm}$ verwendet. In ganzen wurden $11.5 \mathrm{~g}$ Peroxydaseprïparat gewonnen.

$0.2 \mathrm{~g}$ dieses Präparats schieden aus einer Lösung von $2 \mathrm{~g}$ Pyrogallol in $20 \mathrm{ccm}$ Wasser und $20 \mathrm{ccm} 2$-prozentiger Wasserstoffhyperoxydlïsung innerbalb 12 Stunden $0.0296 \mathrm{~g}$ Purpurogallin aus, während ohne Peroxydasen unter denselben Bedingungen nur $0.0116 \mathrm{~g}$ ausgeschieclen worden sind.

Eine weitere Partie von $0.5 \mathrm{~g}$ des P'rüparats wurde abermals wie das Präparat aus Zuckerrübe in $25 \mathrm{~cm}$ Wasser aufgelöst und die Jösung in $80 \mathrm{ccm}$ 20-prozentigen, in Wasser gelösten Pyrogallols in einen Kolben gegossen, welcher wieder an den Apparat zur Prüfung enzymatischer Wirkungen angefügt war, woranf wie bei dem früheren Versuch nach der Methode ron W. Pallatdin vorgegangen wurde. The Resultate sind in den folgenden Tabellen angefübrt.

In 20-prozentiger Pyrugallol-Lüsung.

\begin{tabular}{c|c}
\hline \hline $\begin{array}{c}\text { Dauer des Versuches } \\
\text { in Stunden }\end{array}$ & $\begin{array}{c}\text { Menge des produzierten } \\
\text { CO, in } \mathrm{mg}\end{array}$ \\
\hline & 13.2 \\
16 & 12.6 \\
24 & 0.8 \\
24 & 26.6 \\
64 & 31.4 \\
Nach Hinzufügung von 3-prozentigem $\mathrm{H}_{2} \mathrm{O}_{2}$. \\
24 & 87.3 \\
30 & 29.7 \\
24 & 10.1 \\
24 & 158.5
\end{tabular}

Was die Beziehungen zwischen der Entwicklung der Rübe in verschiedenen Vegetationsperioden, den aus ibnen gewonnenen Mengen von Prroxydasen und ihrer Wirksamkeit anbelangt, so wollen wir uns von den, durch unsere Versuche sichergestellten Tatsachen nicht in weitere Deduktionen vorwageu und behalten uns die Durcharbeitung in dieser Richtung für die nächste Zeit vor.

1) Radieschen hat $\mathrm{Bach}$ bei seinen Versuchen verwendet. 
Wir mőohten es nicht unterlassen, dem hochverehrten Hrn. Prof. Dr. Julius Stoklasa unseren schuldigen Dank abzustatten.

Aus d. Chem.-physiol. Versuchsstation d. böhm. Sektion d. Landeskulturrats für Böhmen an d. Böhm. Techn. Hochscbule in Prag.

\section{A. Wohl:}

Zur Kenntnis der Amido-acetale und Amido-aldehyde.

[Mitteilung aus dem Organ.-chem.Laboratorium der Techn. Hochschule Danzig.]

(Eingegangen am 11. November 1907.]

Vor einiger Zeit ${ }^{1}$ ) habe ich die Ergebnisse dargelegt, die bei der Spaltung des äthylierten Imido-dipropionacetals,

$$
\mathrm{C}_{2} \mathrm{H}_{3} \text {. N }\left[\mathrm{CH}_{2} \text {. } \mathrm{CH}_{2} \text {. } \mathrm{CH}\left(\mathrm{OC}_{2} \mathrm{H}_{5}\right)_{2}\right]_{2} \text {, }
$$

erhalten wurden und die zu einem äthylierten Piperidinaldehyd und seinen Derivaten gefulhrt hatten. Die weitaus schwierigere Aufgabe, die entsprechenden nicht substituierten Verbindungen zu gewinuen, hatte ich zurückgestellt, um an den besser krystallisierenden Stickstoff-substituierten Derivaten erst Erfahrungen über das Verhalten dieser bisher unbekannten Körperklasse zu erwerben. Auf Grund dieser Erfahrungen ist es nun auch gelungen ${ }^{2}$, den eigentlichen Piperidin-aldehyd und Tetrahydropyridin-aldehyd zugänglich zu machen, und von diesem aus den ersten Schritt in ein Gebiet zu tun, dessen AufschlieBung das Ziel der Arbeit bildet, nämlich die Synthese der zum Loiponreste des Chinins in Beziehung stehenden Piperidinderivate.

Nach wesentlich derselben Arbeitsmethode, die bei den äthylierten Verbindungen zur Anwendung kam, kann man bei sehr sorgfältiger Ausarbeitung der Versuchsbedingungen, aus dem Imidodiacetal $\mathrm{HN}\left[\mathrm{CH}_{3} . \mathrm{CH}_{2} . \mathrm{CH}\left(\mathrm{OC}_{2} \mathrm{H}_{3}\right)_{2}\right]_{2}$ in guter Ausbeute das salzsaure Salz des Tetrahydropyridinaldehyds-3,<smiles>O=CC1CCCN1</smiles>

krystallisiert erhalten. Der freie Aldehyd läßt sich vach dem früher heschriebenen Verfahren mittels Kaliumcarbonat und Äther nicht ge-

1) Diese Berichte 88, 4154 [1905].

3) Vergl. die năchstfolgende Abhandlung von $A$. Wobl und M. S. Losanitsch. 